\title{
CONTROLLER DESIGN FOR MAGNETIC LEVITATION SYSTEM USING BACTERIA FORAGING ALGORITHM TECHNIQUE
}

\author{
${ }^{1}$ B. D Halilu, ${ }^{2}$ L. Maijama'a, ${ }^{1}$ A. G Jumba, ${ }^{1}$ S. A Baraza, ${ }^{1}$ A. A Jimoh AND ${ }^{1}$ K.T Aminu \\ ${ }^{1}$ Department of Electrical Engineering Technology, Abubakar Tatari Ali Polytechnic. \\ ${ }^{2}$ Department of Electrical and Electronic Engineering Technology \\ Federal Polytechnic Bauchi.
}

Nigeria

\begin{abstract}
This paper presents the use of Bacteria Foraging Optimization Algorithm (BFOA) to tune Proportional, Integral and Derivative (PID) controller parameters for real time control of Feedback 33-210 Magnetic Levitation System (MLS). The system is hardware in the loop training kit based on MATLAB which gives leverage for controller modification or adaptability. It consists of inbuilt PID and PD controllers. The inbuilt system PID gains were tuned for optimal performance of the real-time system in regulating the ball position and tracking a reference input. Real-time system gave the percentage error of $11.8 \%$ and $33.6 \%$ for the optimal and inbuilt PID controllers respectively. Similarly, real-time system results gave the percentage error of $81.4 \%$ and $128.7 \%$ for optimal and inbuilt PD controllers respectively. Simulation results gave the percentage error of $0.65 \%$ and $23.7 \%$ for the optimal and inbuilt PID controllers respectively. Its PD controller counterpart presents the percentage error of $18.4 \%$ and $336.6 \%$ for optimal and inbuilt simulations scenarios respectively.
\end{abstract}

Keywords: Magnetic Levitation System (MLS), BFOA, PID and PD controllers.

\section{INTRODUCTION}

MLS works on the principle of an object being suspended in the air with no support other than the magnetic fields. The suspended object has no physical contact with the stable part of the system, therefore eliminating friction in the dynamics. This technology minimizes energy loss due to friction which in turn increases efficiency, reduce maintenance costs and increase the life span of the system. Its application has rapidly increased because of its ability to eliminate energy loss due to friction, this include, high speed maglev trains, magnetic bearings which are used in suspending the rotating shaft of turbines, pumps, fans and other rotating machines [7].

Noting the nonlinear, open loop unstable nature of magnetic levitation system, the inbuilt PID controls of the laboratory test bench MLS 33-210 does not exert the optimum force to keep the ball suspended. It either attracts or drops the ball due to excess or insufficient control inputs. The control system therefore needs to be properly tuned.

\subsection{Magnetic Levitation Set Description}

The description of the system setup refers to the mechanical-electrical part and the control aspect. As shown in Figure 1, the maglev system consists of connection interface panel with a mechanical unit on which the coil and infrared sensor are mounted. The package also includes a steel ball which measures $21 \mathrm{~g}$ in mass and $50 \mathrm{~mm}$ in diameter [2]. 


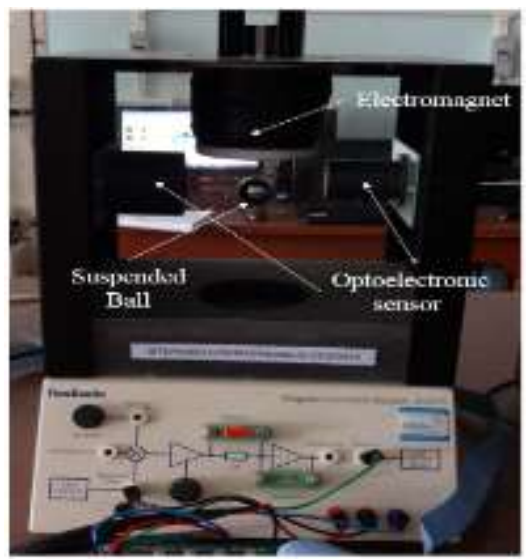

Figure 1: Maglev 33-210 Mechanical Unit.

The optoelectronic sensor determines the vertical position of the ball and passes it to the controller through the Advantech card (PCI17011 I/O Board). Based on the difference between the desired signal and measured output, the controller sends current to the actuator. The actuator is an electromagnet formed by wrapping copper wire of 2850 turns on a high permeability cylindrical iron core. A suitable controller is therefore needed to adjust current through the actuator to stabilize the ball and to make it follow a predetermined trajectory [2].

Apart from the mechanical unit, the electrical unit equally play an important part in MLS control. It serves as the medium through which the measured signal is transmitted to the PC through the I/O board. The Analogue Control Interface (ACI) with model number 33-301 is used to transfer control signals from the PC to the maglev and back through the I/O board. The mechanical and electrical units provide a complete control system setup presented in Figure 2 [2].

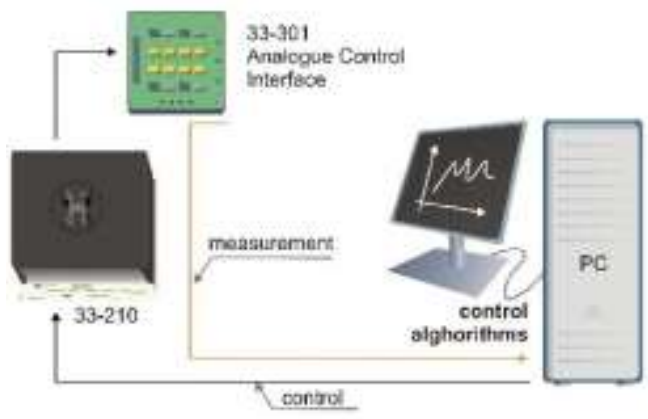

Figure 2: MLS control system setup

\subsection{Literature Review}

\section{a. PID Controller}

PID controller is undoubtedly the most popular controller in industries due to its convenience in design, cost effectiveness and acceptable robustness [8]. It has become an industrial standard because of its simplicity and good performance; however, its performance can still be improved by using better tuning technique to obtain better controller parameters.

PID controller has occupied a dominant position in the industrial process control. Since 1940, there have been many advanced control method, but the PID control is still the best-known industrial process controller because of its simple structure and good robustness in a wide range of operating conditions [10]. Presently, PID control strategy still accounts for about 95\% of all industrial controllers [5].

The control effect of PID controller is mainly depended on three parameters of the controller: proportional coefficient $P$ integral coefficient $I$ and differential coefficient $D$. Designing and tuning the three parameters of PID controller are very important, but it is not easy practical wise [9].

[4] Designed PID controller for unstable SISO (Magnetic Levitation Model) systems in the frequency domain using Neimark Dpartition which ensured desired phase margin of open loop system in addition to stability.

\section{b. Bacterial Foraging Optimization Algorithm as PID Tuning Technique}

BFOA is attracting the attention of researchers recently because of its efficiency in solving real-time optimization problems arising from several application domains. The underlying biology behind the foraging strategy of $E$. coli bacterium is emulated in an extraordinary manner and used as a simple optimization algorithm. 
[6] Showed that the E. coli bacterium performed a kind of sampling and can keep memory of the past, makes comparison with the current with a view of making decision based on the difference. The ability of the $E$. coli bacterium to keep memory which is an additional mechanism that enable it to make comparison and few internal control rules present the algorithm suitable for tuning the parameters of the inbuilt PID controller meant for the position control of the MLS 33-210, since the controller makes comparison and act on the difference between the reference signal and the real ball position. Table 1 present the parameters used for the optimization of PID gains obtained in this work.

Table 1: BFOA Initialization Parameters [1]

\begin{tabular}{|l|l|}
\hline Parameters & Value \\
\hline Dimension of search space $(\mathrm{p})$ & 3 \\
\hline Population size $(\mathrm{s})$ & 10 \\
\hline Chemotactic step $\left(N_{\varepsilon}\right)$ & 4 \\
\hline Length of swim $\left(N_{s}\right)$ & 4 \\
\hline Reproduction step $\left(N_{r \varepsilon}\right)$ & 4 \\
\hline Elimination-dispersal $\left(N_{e d}\right)$ & 3 \\
\hline Reproduction rate $\left(S_{r}\right)$ & $s / 2$ \\
\hline Elimination/dispersal probability $\left(P_{e d}\right)$ & 0.25 \\
\hline
\end{tabular}

\section{MATERIALS AND METHOD}

The materials used for this work includes [2]:

- Maglev 33-210 Mechanical Unit Manufactured by Feedback Inc. Co

- 33-301 Analogue Control Interface

- $\quad$ PC with installed PCI-1711 lab I/O Board

- $\quad$ MATLAB R2015a

- Connecting Leads

\section{a. Real-time PD Control of Ball Position Experiment}

This experiment was carried out to control the ball position using PD controller. It was tested in real-time. Maglev_PD.mdl presented in Figure 3. The PD controller parameters were varied based on the obtained tuned values for the optimal control strategy and the response observed in terms of how well the reference signal was tracked by the system output. Reference signals such as sinusoidal and rectangular signals in addition to the step input were used and the system response which was stored in "To workspace" block was saved and the response plot of the system based on varying reference signals were plotted. For this experiment the integral term $K_{I}$ was set to zero. The MLS 33-210 was connected through the PCI1711 I/O card to the ACI 33-301 as stated in [2].

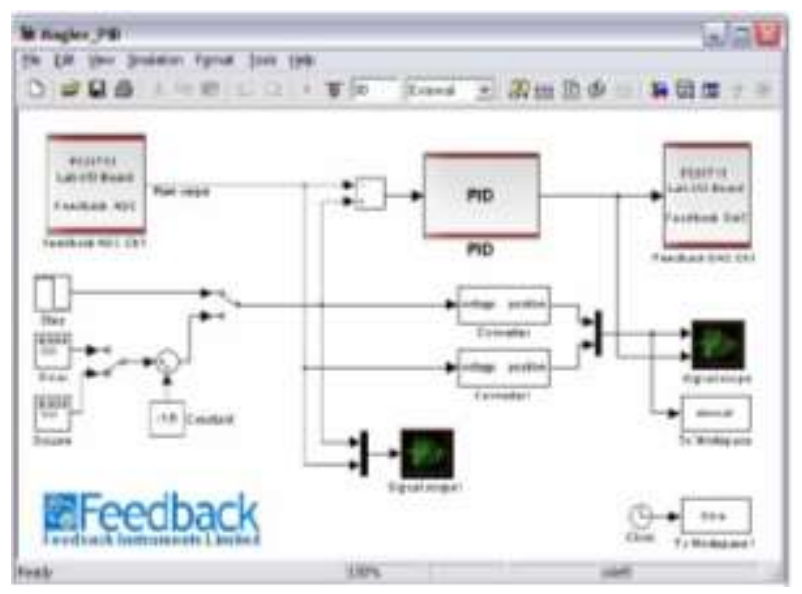

Figure 3: Real-time Maglev Ball Position Control 


\section{b. Real-time PID control of ball position experiment}

The integral action in magnetic levitation can improve the system performance in terms of error minimization. In this experiment Maglev_PID.mdl is presented in Figure 3 with the integral term value as obtained from the inbuilt PID system and the optimal parameter.

\section{RESULT AND DISCUSSION}

\section{a. Optimal and Inbuilt PD Controller Simulation Results}

The performances with the application of PD and PID controllers were evaluated for the non-optimal and optimal conditions. Figures 4 (a), b and c present simulation responses obtained using optimal and inbuilt PD controller. This shows a significant offset resulting in a percentage error of $336.6 \%$ and $18.4 \%$ obtained with inbuilt and optimal PD controllers respectively.

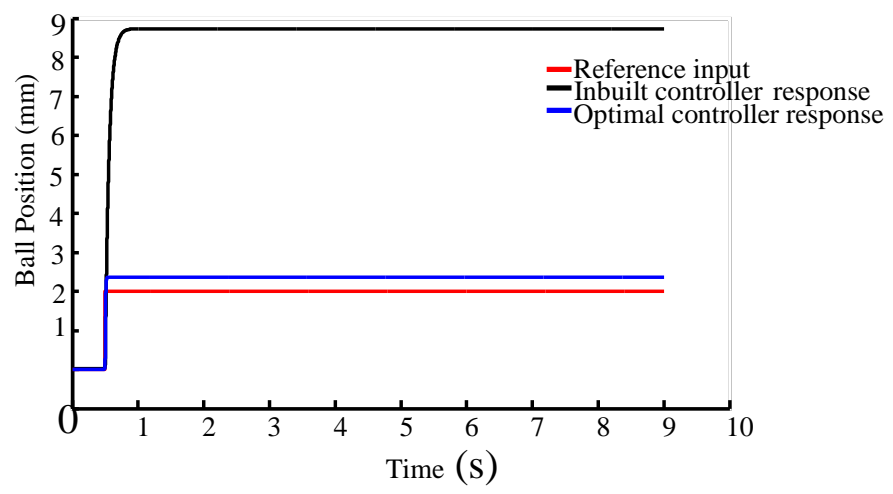

(i): Step input
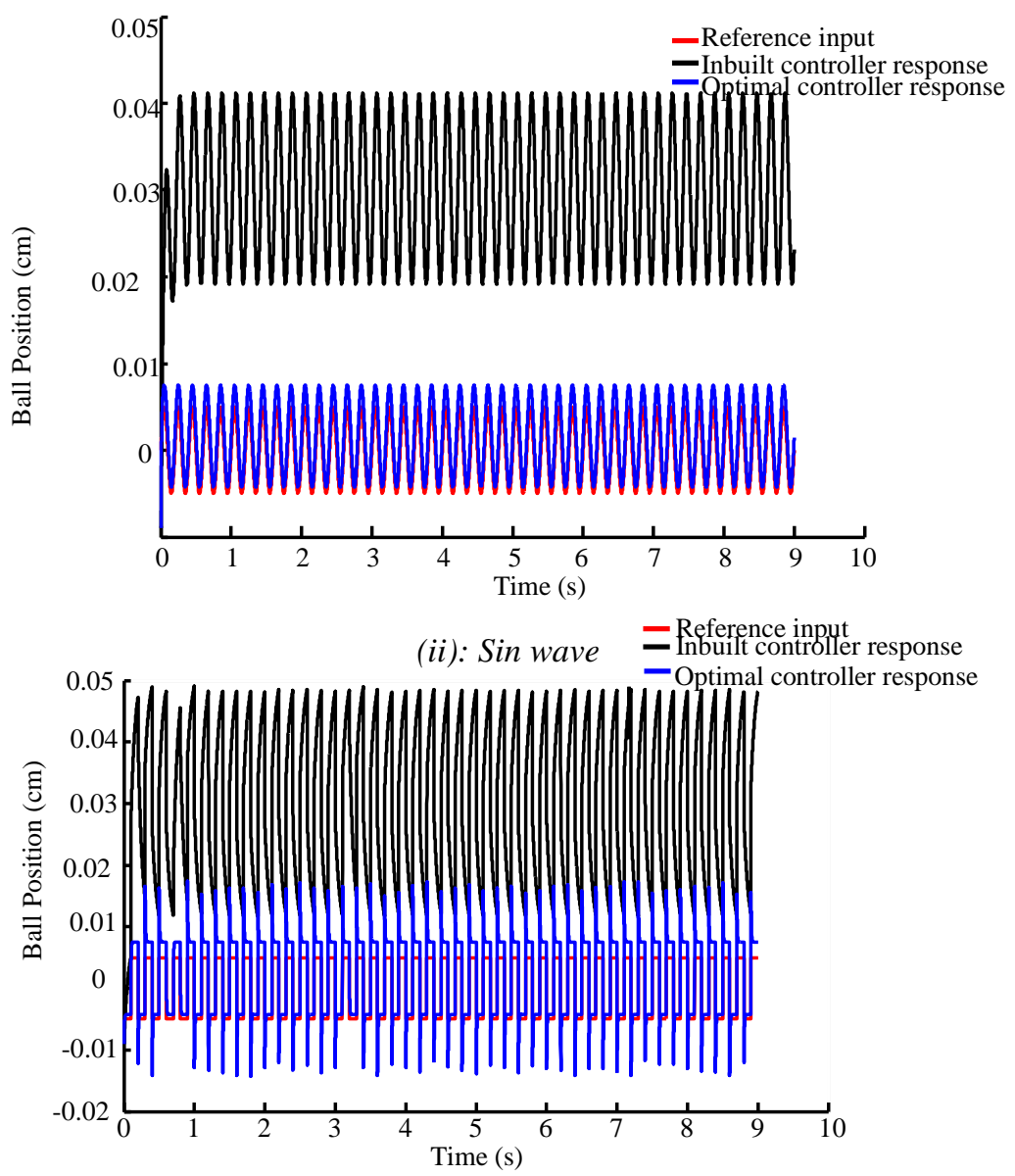

(iii): Square input. 


\section{b. Optimal and Inbuilt PID Controller Simulation Results}

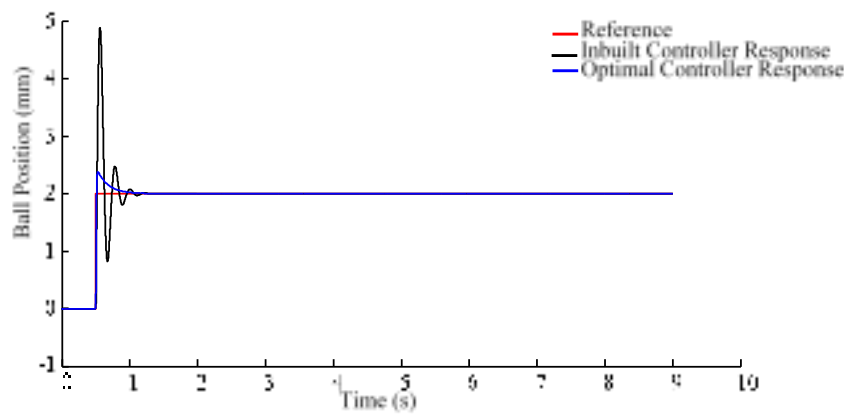

Figure 5: Simulated step input response

Figures 5 show the effect of the optimal gains in tracking the reference signal. The optimal and inbuilt PID controller gives an overshoot of $0.363 \%$ and $2.886 \%$ respectively. The percentage error of $0.65 \%$ and $23.7 \%$ were obtained for optimal and inbuilt PID controllers respectively in simulation scenario.

Figure 6 shows the optimal controller tracking the reference signal (sinusoidal wave) more closely than the inbuilt PID controller.

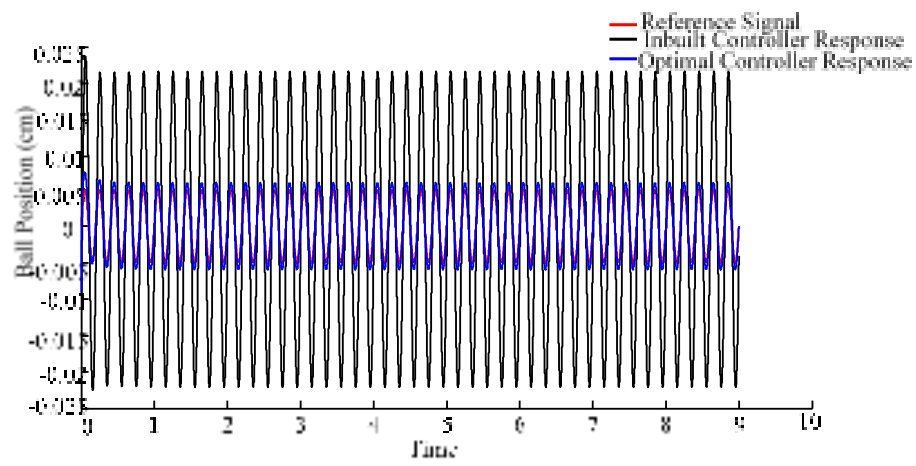

Figure 6: Simulated sine wave input response

Table 2 gives the values obtained from the response plot in Figures 4 and 5 using the step input response. For a set point of $0.02 \mathrm{~cm}$ in simulation mode, the measured ball position gave the values of $0.02012 \mathrm{~cm}$ and $0.02474 \mathrm{~cm}$ for optimal and inbuilt PID controllers respectively. This in turn resulted in percentage error of $0.65 \%$ and $23.7 \%$ respectively. For the same set point of $0.020 \mathrm{~cm}$ in real time mode, the measured ball position values were obtained as $0.02368 \mathrm{~cm}$ and $0.08735 \mathrm{~cm}$ for optimal and inbuilt PD controllers respectively resulting in the percentage error of $18.4 \%$ and $336.6 \%$. Though the result shows that BFAO tuned PD and PID controllers performs better than their inbuilt counterparts, optimal PID controller performs significantly better than the optimal PD controller.

Table 2: Ball Position Simulation

\begin{tabular}{|c|c|c|c|c|}
\hline & \multicolumn{2}{|c|}{ PID Controller } & \multicolumn{2}{c|}{ PD Controller } \\
\hline Ball Position (cm) & Optimal & Inbuilt & Optimal & Inbuilt \\
\hline Reference & 0.020 & 0.020 & 0.020 & 0.020 \\
\hline Measured & 0.02012 & 0.02474 & 0.02368 & 0.08735 \\
\hline Error (\%) & 0.65 & 23.7 & 18.4 & 336.6 \\
\hline
\end{tabular}

\section{c. Optimal and Inbuilt PD Controller Real-time Results}

The plant performance based on the PD controller was evaluated for the inbuilt and optimal conditions. Figures 7 (i), (ii) and (iii) show an improved performance in terms of reducing the off-set by the optimal PD controller in comparison to the inbuilt PD controller on the MLS 33-210. 

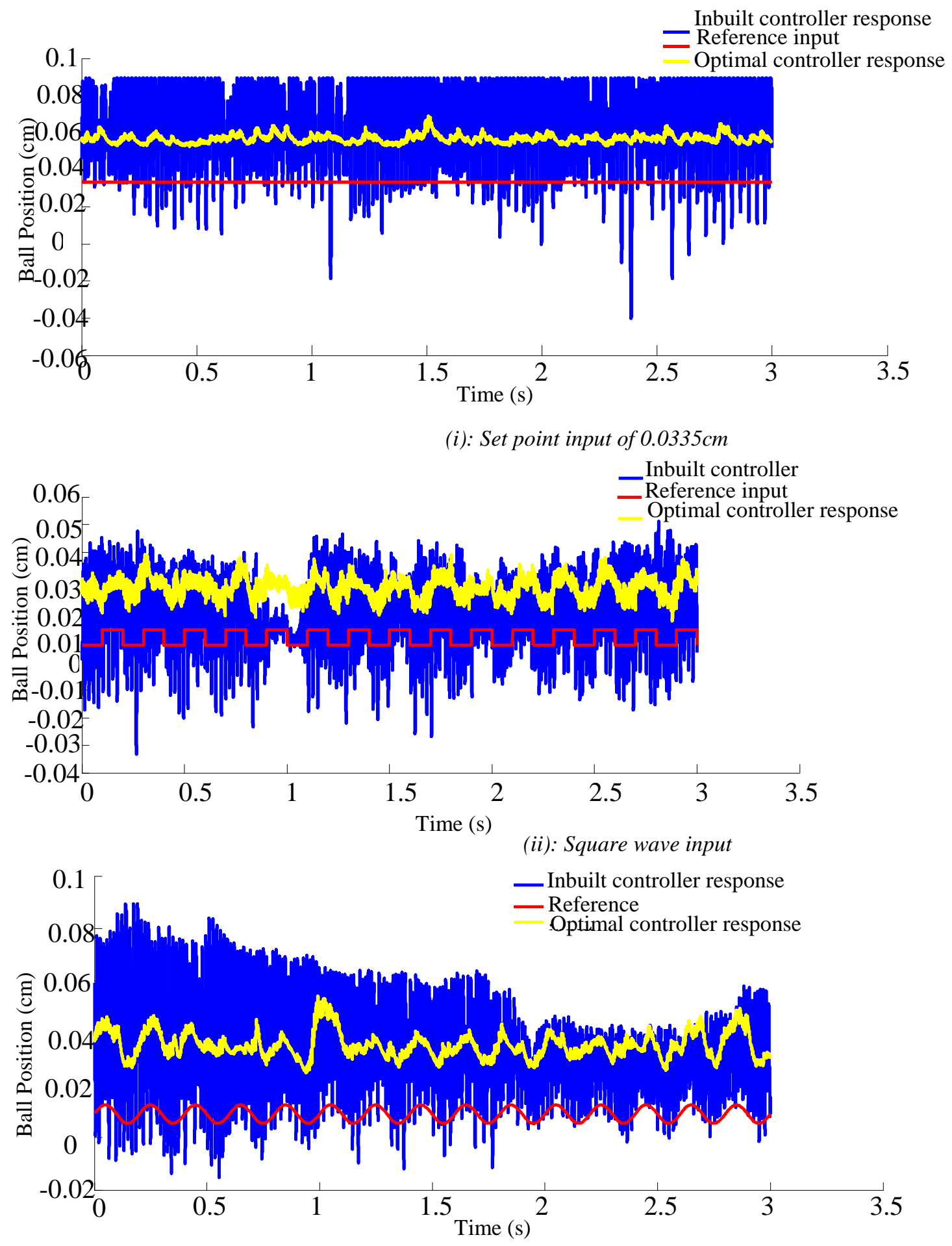

(iii): Sin wave input

Figure 7:Real-time System Response with PD Controller

\section{d. Optimal and Inbuilt PID Controller Real-time Results}

Figures 8 (i), (ii) and (iii) show the real-time responses for inputs-set point regulation, tracking of square and sine waves respectively. 


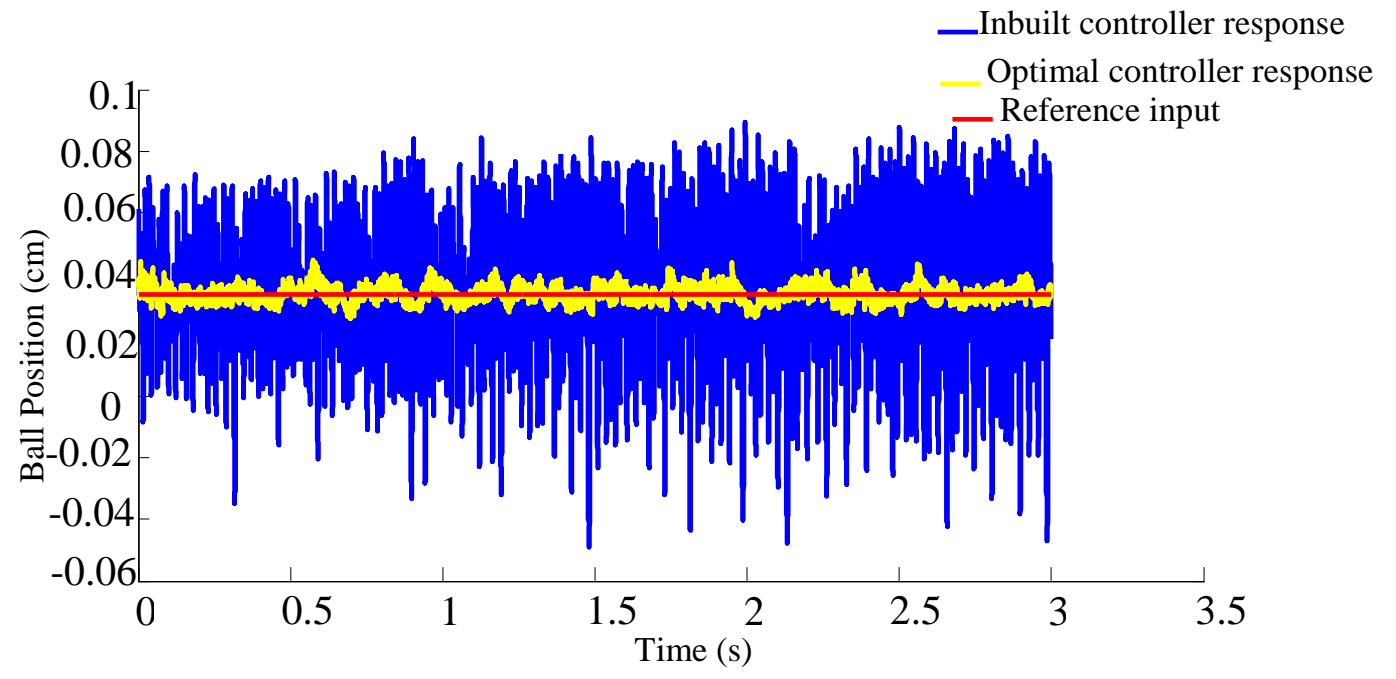

(i): Set point input of $0.0335 \mathrm{~cm}$
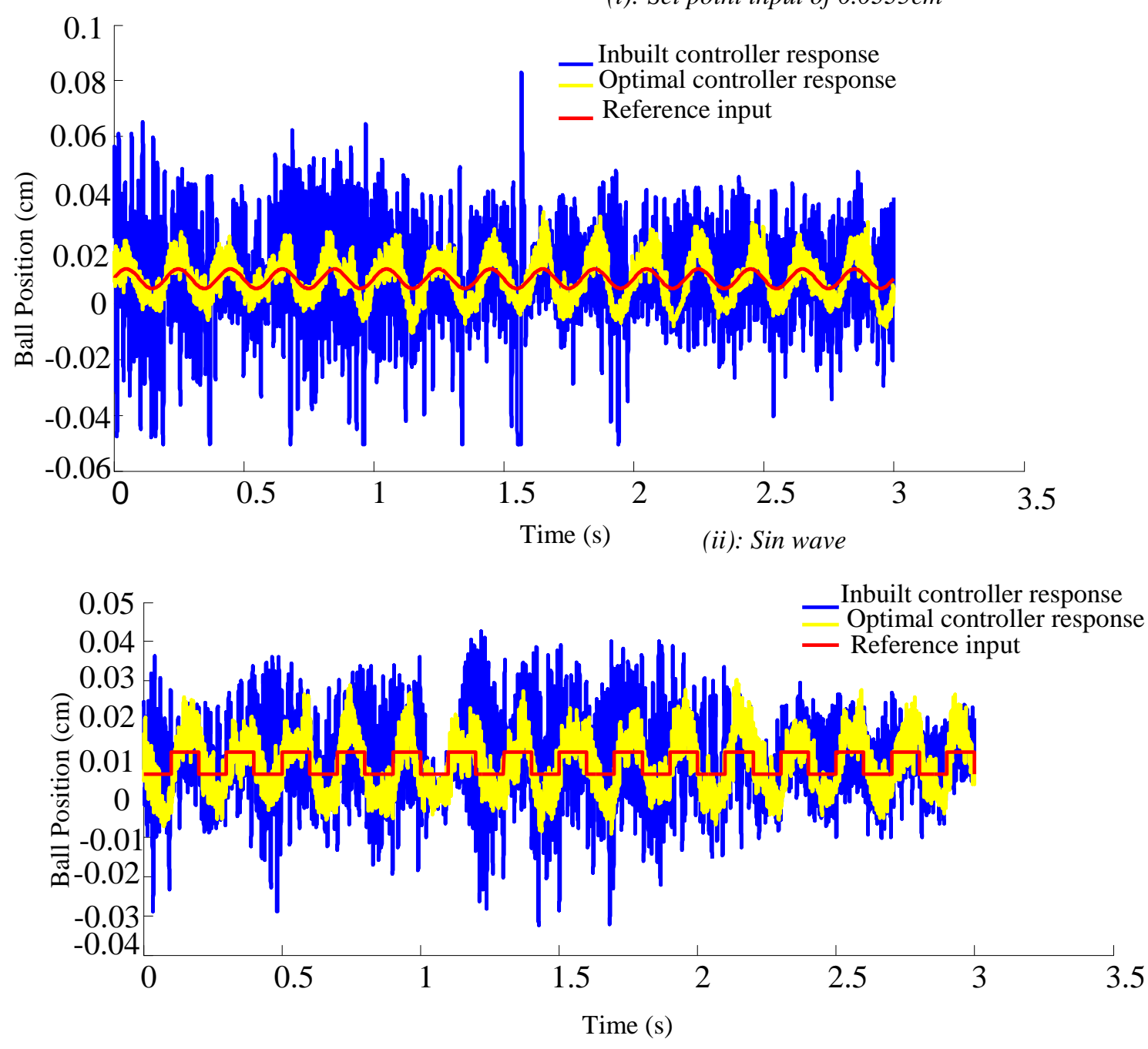

(iii): Square wave

Figure 8:Real-time System Response with PID Controller

From Table 3, for a set point of $0.0335 \mathrm{~cm}$ in real-time, the measured ball position gave the values of $0.03744 \mathrm{~cm}$ and $0.04474 \mathrm{~cm}$ for optimal and inbuilt PID controller parameters respectively. Also, measured values of $0.06078 \mathrm{~cm}$ and $0.07668 \mathrm{~cm}$ were obtained with optimal and inbuilt PD controllers respectively. The optimal and inbuilt PID controller gives a percentage error of $11.8 \%$ and $33.6 \%$ respectively. For optimal and inbuilt PD controller, the percentage error of $81.4 \%$ and $128.7 \%$ were obtained respectively. The Result shows that optimal PID controller performs better than its PD counterpart. 
International Journal of Advances in Scientific Research and Engineering (ijasre), Vol 5 (5), May-2019

Table 3: Real-time Ball Position

\begin{tabular}{|c|l|l|l|l|}
\hline & \multicolumn{2}{|c|}{ PID Controller } & \multicolumn{2}{c|}{ PD Controller } \\
\hline Ball Position (cm) & Optimal & Inbuilt & Optimal & Inbuilt \\
\hline Reference & 0.03350 & 0.03350 & 0.03350 & 0.03350 \\
\hline Measured & 0.03744 & 0.04474 & 0.06078 & 0.06078 \\
\hline Error (\%) & 11.8 & 33.6 & 81.4 & 128.7 \\
\hline
\end{tabular}

\section{CONCLUSION}

In this paper the inbuilt PD and PID controller gains of MLS 33-210 manufactured by Feedback Inc. were tuned using BFOA and analysis made in terms of percentage error which has direct effect on the ball levitation at the desired position as well as tracking a predetermined trajectory. The performance of the inbuilt and optimized PID and PD controllers were compared. The optimal PID controller gives a better performance in comparison to the optimal PD Controller. It reduces the percentage error by $69.6 \%$ and $17.7 \%$ in real-time and simulation scenarios respectively. This led to better tracking of the predetermine trajectories hence positioning of the ball at the desired position. Result from this paper therefore present BFOA as a better tuning technique for the PID and PD controller parameters of MLS 33-210.

\section{REFERENCES.}

[1] B.D Halilu, E.C Anene, E.E Omigzegba, L. Maijama'a and S.A Baraza (2019). Optimization of PID Controller Gains for Identified Magnetic Levitation Plant Using Bacteria Foraging Algorithm. IJEMT Vol. 5 No. 1 pp12-18.

[2] Feedback Instruments Ltd User Manual (2006). Magnetic Levitation Control Experiments Manual. 33-942S Ed01 122006, Feedback Part No. 1160-3394S.

\section{[3] http://www.feedback-intruments.com/products/}

[4] Hypiusova, M. and Osusky, J. (2010): PID Controller Design for Magnetic Levitation Model. Paper presented at the International Conference Cybernetics and Informatics, VYSNA BOCA, Slovak Ruplic. Pp1-7

[5] Kang J., Meng W., Abraham A. and Liu, H. (2014). An adaptive PID neural network for complex nonlinear system control. Journal of Neurocomputing, 135 79-80.

[6] Kevin, M. P. (2010): Bacteria Foraging Optimization: International Journal of Swarm Intelligence Research. 1(1), 1-16.

[7] Minhaj, A., Fahad, M. H., Emdadul, M. H., Omar, F. and Mohammed, M. (2016). Design and Construction of a Magnetic Levitation System Using Programmable Logic Controller. American Journal of Mechanical Engineering. 4(3), 99-107.

[8] Prasanta, R., Manashita, B., Lalbahadur, M. and Nikita, S. (2015): Design and Implementation of FOPID Controllers by PSO, GSA and PSOGSA for MagLev System. Paper presented at the International Symposium on Advanced Computing and Communication. 10(3) 2009-2011.

[9] Rongrong, S. and Zili, C. (2014). Design of PID Controller for Maglev System Based on an Improved PSO with Mixed Inertia Weight. Journal of Networks. 9(6), 100-112.

[10] Xie, F., Hu, J. and Chen, P. (2012). Research on the parameter optimization of PID controller. Advances in Information Sciences and Service Sciences. 4(2), 267-274. 